\title{
The Expressive and the Receptive One Word Picture Vocabulary Test (EOWPVT \& ROWPVT). (A combine pilot study in Greek school aged children \& data for expressive and receptive language for this population)
}

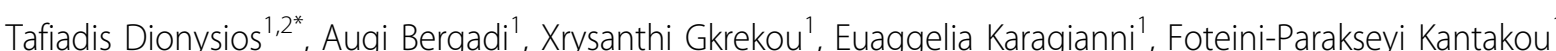
Kaliopi Kentioglou', Iosifina Paroutiadou', Konstantina Papageorgiou', Maria Tafiadi ${ }^{3}$, Georgia Zafeiropoulou ${ }^{1}$

From $1^{\text {st }}$ International Congress on Neurobiology and Clinical Psychopharmacology

and European Psychiatric Association Conference on Treatment Guidance

Thessaloniki, Greece. 19-22 November 2009

\section{Background}

The present pilot study was the adaption of receptive and expressive language tests' (ROWPVT and EOWPVT) for Greek children aged from 6 years till 11 years and 11 months, and to locate any differences between receptive and expressive language.

\section{Materials and methods}

The commercial versions of the tests were adapted in Greek language by a linguist, three speech language therapists and 2 native speakers of Greek language, having proficiency in English, and two native speakers of English having proficiency in Greek, and changes were contacted, for the best representation of the Greek version. In this research took part 615 participants (m: 310, f: 305) recruited from Greek Schools. The sample was independent from origin and socio - economic situations. Also an ENT, neurological and a psychological examination were also requested, so no medical problems could probably influence the test results.

\section{Results}

Statistical analysis of the data revealed that the results obtained are generally consistent to other results reported. No statistically significant differences were found

${ }^{1}$ Department of Speech and Language Therapy, T.E.I. of Epirus, Ioannina, loanninon, Greece according to sex. Also reliability and validity test were contacted and showed high criterion $(\mathrm{a}-$ Chronbach $=$ $.823, \& .834)$.

\section{Conclusions}

The test appears to be sensitive for school aged Greek population and presents satisfactory criterion, internal consistency, temporal stability, interrater reliability, high content validity. The participants demonstrated clear patterns of responses but in some ages differences were located between expressive and receptive language. Further research must be done, to exclude or include any adaptation, learning or developmental reasons.

\section{Author details}

'Department of Speech and Language Therapy, T.E.I. of Epirus, loannina, Ioanninon, Greece. ${ }^{2}$ Department of Neural Sciences and Sensory Organs (School of medicine), University of Ioannina, Ioannina, loanninon, Greece. ${ }^{3}$ Department of Mathematics, National and Kapodistrian University of Athens, Athens, Attika, Greece.

Published: 22 April 2010

doi:10.1186/1744-859X-9-S1-S193

Cite this article as: Dionysios et al:: The Expressive and the Receptive One Word Picture Vocabulary Test (EOWPVT \& ROWPVT). (A combine pilot study in Greek school aged children \& data for expressive and receptive language for this population). Annals of General Psychiatry 2010 9(Suppl 1):S193. 